\title{
Formación docente para la educación a distancia: la construcción de las competencias docentes digitales
}

\section{Formação docente para a educação a distância: a construção de competências docentes digitais}

\section{Teacher training for distance education: the construction of digital teaching skills}

\author{
Isamélia Santos Guimarães Carvalho ${ }^{1}$ \\ José Tejada ${ }^{1}$ \\ Katia Verónica Pozos Pérez ${ }^{1}$
}

DOI: http://dx.doi.org/10.20435/serie-estudos.v24i51.1296

\begin{abstract}
Resumen: La formación docente es imprescindible en la Educación a Distancia y debe estar planificada de acuerdo con las necesidades formativas del profesorado y las demandas de la sociedad digital, posibilitando al profesor la construcción de las competencias docentes digitales. Plantear la formación docente en EaD, permite reconocer una nueva realidad y nuevos modelos educativos, rompiendo con antiguos paradigmas hacia una práctica pedagógica reflexiva y autónoma. En ese artículo analizamos las competencias digitales del perfil del profesor de educación superior en sus distintos niveles con el objetivo de establecer las necesidades formativas y planificar una formación docente para los referidos profesores. De acuerdo a dicho perfil docente se realiza un estudio cualitativo, de evaluación de las competencias digitales docentes, implicando las voces de los protagonistas (profesores, directivos, estudiantes) y se establecen las diferentes necesidades formativas, que dan pie a la propuesta formativa.
\end{abstract}

Palabras clave: educación a distancia; formación docente; competencias docentes digitales.

Resumo: A formação docente é imprescindível na Educação a Distancia e deve estar planejada de acordo com as necessidades formativas do professorado e as demandas da sociedade digital, possibilitando ao professor a construção das competências docentes digitais. Planejar a formação docente em EaD, permite reconhecer uma nova realidade e novos modelos educativos, rompendo com antigos paradigmas para uma prática pedagógica reflexiva e autônoma. Neste artigo analisamos as competências digitais do perfil do professor da educação superior em seus diferentes níveis com o objetivo de estabelecer as necessidades formativas e planejar uma formação docente para os referidos professores. De acordo com este perfil docente se realiza um estudo qualitativo, de avaliação das competências digitais docentes, implicando as vozes dos protagonistas (professores e diretores) e se estabelecem as diferentes necessidades formativas, que darão forma a proposta formativa.

\footnotetext{
${ }^{1}$ Universitat Autònoma de Barcelona, Barcelona, Espanha.
} 
Palavras-chave: educação a distância; formação docente; competências docentes digitais.

Abstract: Teacher training is essential in distance education and must be planned according to the teachers' training needs and the demands of the digital society, enabling the teacher to build digital skills. Raising the teacher training in distance education, allows to recognize a new reality and new educational models, breaking with old paradigms towards a reflexive and autonomous pedagogical practice. In this article, we analyze the digital skills of the higher education teachers with the objective of establishing the training needs and planning a teacher training for the teachers. According to this teaching profile, a qualitative study of the evaluation of the digital teaching competences is carried out, involving the voices of the protagonists (professors and manager) and establishing the different training needs, which give rise to the training proposal.

Keywords: distance education; teacher training; digital teaching skills.

\section{INTRODUCCIÓN}

Los procesos educativos y tecnológicos se aceleran de manera desenfrenada, muchas veces en sentidos opuestos, pero todos se enfrentan a la necesidad de una "unión" o "fusión". Muchos estudiosos e investigadores de los fenómenos educativos y sociales consideran estas transformaciones necesarias e importantes, pero debemos tener la capacidad de seleccionar de manera reflexiva, responsable y coherente los entornos y las herramientas tecnológicas adecuadas a partir de las finalidades educativas.

La educación trabaja con valores éticos y principios que no pueden ser descuidados al pensar y construir una sociedad digital. Estos valores son referentes e imprescindibles al planear el proceso de integración y utilización de la tecnología a la educación y la formación docente. Siendo así, la tecnología, la consistencia del discurso pedagógico y la formación docente deben caminar juntos y en total consonancia.

Así pues, la formación del profesor y, por consiguiente, la del alumno que se va a desarrollar e integrar en esta sociedad digital es muy importante, una vez que todo el proceso de creación y construcción de nuevos contenidos y herramientas tecnológicas, deben estar integrados y fundamentados en una visión sólida de la educación. Aun cuando la tecnología se ha convertido en un aspecto de gran valor en nuestra sociedad, que suscita necesidades y demandas crecientes, los sistemas educativos no han logrado aún responder adecuadamente a ellas, y, por consiguiente, su responsabilidad social de construir estas respuestas es cada vez más necesaria y urgente. 
De la misma forma en que se integren las tecnologías en la función docente, es también necesario integrarlas de manera transversal en cada uno de los roles y funciones que desempeña el profesorado, es decir, en su desarrollo profesional, para que también sirvan como una herramienta de apoyo para potenciar sus funciones de gestión e investigación en la institución, así como las de responsabilidad social con el entorno, entre otras. Con ello se deriva, por tanto, la necesidad de que el profesorado desarrolle nuevas competencias digitales asociadas a cada uno de sus roles profesionales que son necesarios para una educación en la Sociedad del Conocimiento. (POZOS, 2015, p. 35).

No podemos negar la importancia del constante diálogo entre la Educación y la tecnología, donde esa última puede contribuir de manera inestimable para la transformación de los métodos de enseñanza, la formación docente y el aprendizaje de los alumnos. Sin embargo, es importante resaltar que la Tecnología no es necesariamente más relevante o eficaz que los métodos de enseñanza tradicionales, pero son necesarias y están cada vez más presentes en la vida cotidiana y forman parte del universo de los jóvenes, así que es inminente la necesidad de su integración a la educación.

No obstante, hay algunas preguntas acerca del currículo escolar y de la tecnología que nos hace falta reflexionar y son de extrema importancia para la integración adecuada de las tecnologías en la educación ¿¿ qué forma la tecnología puede contribuir a la educación, con tantos contenidos académicos, distantes de la realidad del alumno? ¿Cómo crear herramientas pedagógicas que se adapten a las especificidades de cada contenido o área de conocimiento, así como su eficacia para el aprendizaje del sujeto? Estas son algunas cuestiones que, muchas veces, nos preguntamos y las respuestas cuestan, son preguntas que nos hace reflexionar y necesitamos profundizar.

Para que estos contenidos se adapten a los entornos presenciales, semipresenciales y virtuales es necesario que sean previamente seleccionados y elaborados, considerando los cambios y redefiniciones en su forma de presentación, construyendo mensajes que potencien las virtudes tecnológicas, concibiendo las metodologías y estrategias de la enseñanza que posibiliten al máximo el aprendizaje, eso significa liberarse de las metodologías tradicionales, basada en un discurso científico lineal, cartesiano y positivista.

La educación a distancia se consolida y nos enseña una nueva concepción y posibilidades acerca de la educación y del aprendizaje. Ratificamos que las 
distintas herramientas tecnológicas utilizadas en los espacios virtuales necesitan estar vinculadas a la pedagogía con el objetivo de ampliar y mejorar la práctica docente (SANCHO; HERNÁNDEZ, 2006). Así, es inminente la necesidad de invertir en la formación de profesores, sobre todo los que actúan en los entornos virtuales y lidian diariamente con nuevas formas de aprendizaje y también con un nuevo perfil de alumno.

\section{LA FORMACIÓN DOCENTE}

La educación tiene un valor decisivo para la sociedad de la información y del conocimiento, está en un punto estratégico y de gran importancia. En cambio, la formación docente tiene un valor único para la educación a distancia, puesto que es un camino para la desconstrucción de la idea instrumental de la tecnología y asegurar su función pedagógica (ZABALZA, 2007). Conviene subrayar que la escuela y la educación formal, siempre han privilegiado la escritura, resistiendo a la interacción con las herramientas tecnológicas, considerando apenas su carácter instrumental y técnico. De ahí que la formación se hace necesaria e imprescindible.

El proceso de construcción de las competencias digitales debe estar vinculado a las competencias docentes, trabajando de manera transversal, posibilitando al profesor asumir una nueva postura en su práctica pedagógica, en su actuación social y colectiva. Aunque la formación del profesor sea un proceso individual con aspectos individuales y peculiares, respecto a la subjetividad del sujeto, no podemos olvidar que es un proceso por lo cual se supone una transformación social. Por tanto, ese es un gran desafío para la formación docente y para la educación, favorecer la construcción del conocimiento y la adquisición de las competencias docentes digitales permitiendo al profesor elegir, gestionar y crear nuevas posibilidades de acceso al conocimiento.

En efecto, la formación docente es un proceso permanente y continuo, condición sine qua non para la construcción de las competencias docentes digitales, para el aprendizaje significativo de los alumnos y una clave para el desarrollo profesional. Eso vale para los distintos entornos educativos, es decir, presencial, semipresencial y virtual, la formación es un desafío para el profesor, permite que se rompa con antiguos paradigmas educativos y además demanda una postura reflexiva acerca de su práctica pedagógica, sobre todo en los entornos virtuales. 
La formación docente en la educación superior es escasa, un problema real y actual, puesto que, es laborioso planificar una formación a partir de múltiples aproximaciones disciplinarias con profesionales provenientes de diversas áreas de conocimiento. En ese sentido se ratifica la necesidad de políticas públicas para todos los ámbitos educativos, incluso y principalmente en la educación a distancia, dado que aún hay un cierto recelo acerca de las nuevas formas y espacios de aprendizaje.

Pensar y plantear alternativas de formación docente para las instituciones de Educación Superior, permite reconocer una nueva realidad educativa, posibilitando la construcción de nuevos modelos educativos, más flexibles y contextualizados, pertinentes y relevantes para una educación que favorezca la integración de los distintos saberes del mundo y fomente los cambios sociales. Según Ferrari (2012), la competencia digital del docente es indispensable y supera la alfabetización digital en extensión y profundidad.

La Organización de las Naciones Unidas para la Educación, la Ciencia y la Cultura (UNESCO), en su informe de 2008, ha incorporado la competencia comunicativa, es decir, la aptitud de los profesores para mantener contacto con los alumnos, expertos o colegas para compartir ideas, conocimientos y experiencias que enriquezcan el proceso educativo.

Estamos en un momento transformador para la educación, somos privilegiados de poder organizar, gestionar, pensar y reflexionar. Beneficiarse de los inúmeros recursos educativos digitales permitirá a los docentes presentar a sus alumnos infinitas oportunidades, antes impensable, para asimilar los distintos contenidos.

La educación a distancia viabiliza la capacidad de transformar y propone posibilidades de intervenciones eficaces y creativas, permitiendo la creación y gestión del conocimiento, así como, su difusión y aprovechamiento cooperativo. Igualmente, atiende a los objetivos de la educación revelándose como una herramienta de transformación y cambio social. Así, es incuestionable su relevancia y significatividad en la sociedad, pero demanda una formación previa y eficaz.

La preparación y emancipación profesional del docente para elaborar crítica, reflexiva y eficazmente un estilo de enseñanza que promueva un aprendizaje significativo en los alumnos y logre un pensamiento-acción innovador, trabajando en equipo con los colegas para desarrollar un proyecto educativo común. (FERNÁNDEZ, 1997 apud MEDINA, 2006, p. 11). 
Aún en su informe de 2008, la Organización de las Naciones Unidas para la Educación, la Ciencia y la Cultura (UNESCO), certifica que la competencia digital del profesor debe estructurarse en tres fases: competencias básicas, de profundización y de generación del conocimiento, que son parte de un proceso en el que el profesor profundiza el conocimiento y la utilización de las TIC en la medida en que se las va integrando gradualmente de manera significativa, crítica y reflexiva en su práctica docente. Eso conlleva a una a revisión íntegra de los currículos educativos y los procesos de integración de la tecnología en los centros educativos, así como los proyectos de formación planificados y llevados a cabo.

El valor de la educación a distancia no está representado únicamente por la tecnología, sino en el sentido que el profesor le dará, es decir, los procedimientos y metodologías pedagógicas empleados por el docente les atribuyen significado, lo que supone que el profesor esté con sus competencias docentes digitales adquiridas y en proceso de desarrollo constante.

La competencia digital clave del futuro profesorado, entendida como capacidad de realizar actos profesionales con resultados reconocibles en el mundo de la profesión docente, es "saber utilizar e incorporar adecuadamente en las actividades de enseñanza-aprendizaje, las tecnologías de la información y la comunicación. La formación del profesorado no puede reducirse a la adquisición de competencias digitales o destrezas tecnológicas per se sino que debe basarse en su aplicación didáctica. Con carácter general el docente ha de planificar, impartir, tutorizar y evaluar acciones formativas, elaborando y utilizando medios y recursos didácticos, promoviendo la calidad de la formación y la actualización didáctica. (TEJADA, 2009).

Visto que la educación a distancia es un imperativo social e inevitable en el sistema educativo, hace falta un marco referencial y común respecto a la formación docente y a la correcta integración de la TIC con el objetivo de desarrollar la cultura digital en conformidad con la educación. Esa afinidad es un referente importante en el momento de construir y desarrollar un plan de formación consistente y coherente considerando los antecedentes e indicadores valorables, posibilitando estructurar y enlazar la formación y el desarrollo profesional. 


\section{METOdología}

En el presente artículo utilizaremos un diseño de investigación cualitativa aplicando la metodología Historias de vida (CORTÉS, 2011), que se marca dentro de una investigación más amplia en el contexto de las universidades catalanas privadas.

El abordaje cualitativo de este estudio nos ha llevado a analizar casos específicos de la muestra con profundidad, comprendiendo de manera más subjetiva, descubriendo el significado en las acciones socioeducativas. Elegimos el método Historias de vida, dado que nos presenta una riqueza inagotable de materiales, contenidos e innumerables posibilidades de profundizar y articular con el entorno investigado, contemplando las peculiaridades y especificidades del tema.

El en recorrido de nuestra investigación hicimos tres entrevistas en profundidad con cada profesor, los encuentros eran semanales, lo que posibilitaba una reflexión acerca de los temas discutidos en el encuentro anterior, los profesores apuntaban sus reflexiones las cuales discutíamos en el encuentro siguiente, ha sido un proceso con mucha riqueza de información y contenidos.

Siendo así, en la metodología historias de vida, seleccionamos algunas entrevistas a partir de criterios establecidos, tras el análisis y el estudio de los datos y perfil de los profesores, El análisis de los datos fue a través de un estudio de caso minucioso y detallado, con categorías previamente discutidas y elegidas.

\section{DESARROLLO DEL TRABAJO DE CAMPO}

\subsection{Acceso al campo}

El acceso al campo se llevó a cabo durante los meses de septiembre de 2017 a julio de 2018 de manera exitosa, estando en contacto con los aspectos y elementos del contexto de las experiencias de los profesores y su práctica pedagógica. Hicimos una observación diaria del grupo de profesores de 1 universidad con el objetivo de interpretar y entender las relaciones establecidas entre ellos y de qué manera influya en su práctica pedagógica y desarrollo profesional.

\subsection{Protocolo de actualización - institución}

El acercamiento formal, posibilitó la observación y construcción de esquemas sociales, características personales y profesionales, organigramas de 
funcionamiento, identificación de los informantes claves, etc., comprobando las características y perfil de las instituciones están en consonancia con el tema de la investigación y sus objetivos.

\subsubsection{Población-muestra}

La población está formada por profesores universitarios pertenecientes a una universidad privada en Catalunya. Consideramos adecuado seleccionar el muestreo intencional, eligiendo los profesores con distintas formaciones y edades. Elegimos el muestreo intencionado, donde los criterios de representatividad y sus significados están en el discurso, respondiendo a las preguntas de la investigación, posibilitando conocer e interpretar el fenómeno estudiado con profundidad en sus distintas perspectivas. Los profesores investigados vienen de distintas formaciones iniciales, tienen distintas edades y ejercen la función de docente hace muchos años.

\subsubsection{Instrumento}

El instrumento de recogida de datos fue desarrollado y construido contemplando las demandas y cuestionamiento referentes al estudio propuesto. Empleamos la metodología cualitativa, utilizamos un guion para las entrevistas individuales con los profesores a través del relato oral. El objetivo central de las entrevistas en profundidad utilizando el método Historias de Vida es obtener una información más clara, personalizada y subjetiva de los profesores tales como acontecimientos vividos, creencias, valores y costumbres relacionados con el proceso.

\section{RESULTADO Y CONCLUSIONES}

Como hemos comentado anteriormente, dentro de la metodología historias de vida, seleccionamos el relato del director de la institución y dos profesores de esa misma institución. Los profesores fueron seleccionados a partir de criterios establecidos con el objetivo de establecer un contraste entre sus testimonios y recorridos profesionales. Además, elegimos los géneros masculino y femenino, vale resaltar que ambos profesores tienen necesidades formativas, pero desde perspectivas distintas debido a sus diferentes formaciones, experiencias académicas y profesionales. 
En ese sentido, para establecer los resultados seleccionamos algunos momentos de sus relatos en que observamos evidencias acerca de algunas categorías previamente establecidas:

- Formación inicial / Docencia/Necesidades formativas/Resistencia a la tecnología/ Adquisición de las Competencias digitales y Pedagógicas/ Implicaciones de la institución en ese proceso.

Por cuestiones éticas y de confidencialidad no citaremos el nombre de la Universidad la cual realizamos la investigación y utilizaremos la siguiente nomenclatura en cuerpo del análisis: Directora para la Directora académica de dicha institución (género femenino) y Profesores $A$ y B, masculino y femenino respectivamente, para los profesores entrevistados en nuestra investigación.

En el relato del directivo observamos que hay una implicación y un compromiso con el proceso pedagógico, la formación docente y la utilización de la tecnología desde una perspectiva pedagógica, crítica y reflexiva. Cuando le preguntamos acerca de su proceso de adquisición de las competencias digitales y de qué manera eso se refleja en el ámbito institucional, está claro que hay un distanciamiento entre su postura y las decisiones administrativas e institucionales que no son del ámbito académico.

Bueno para mí, sigue siendo un continuo aprendizaje, porque es verdad que yo vengo de otra época yo utilizo mis competencias digitales por las herramientas digitales en la clase de una manera mucho más intuitiva, como un insider room, porque es verdad que he hecho algunas formaciones, algunos pequeños cursos que las universidades ofrecen, principalmente en mi época de doctorado. Pero desde mi punto de vista son cursos muy cortos, muy breves, que te dan pinceladas muy rápidas para saber cómo utilizar una determinada herramienta. Para mí esas formaciones son muy técnicas y muy breves, te enseñan la herramienta y punto. Y yo creo, desde mi punto de vista, que sería importante aparte de aprender a utilizar las diversas herramientas que existen, relacionarlas en el contexto académico y utilizarlas en la clase. (Directora)

Así pues, observamos que la demanda formativa y la de adquisición y desarrollo de las competencias docentes digitales es una necesidad inminente y urgente. Aunque la directora tenga una orientación pedagógica en su gestión, que se supone ser reflexiva y participativa, la misma admite que utiliza la tecnología de manera técnica y que intenta vincularla a su práctica docente de manera intuitiva. 
Yo lo que siento en mi trayectoria es que la utilizo como una forma técnica, más que como una forma realmente de vincularla con la función pedagógica. Intento realmente hacerlo, pero desde una forma... una forma intuitiva. (Directora)

En todas las entrevistas observamos los términos "Utilización de manera intuitiva" o como "Autodidacta", es decir, ningún profesor tuvo una formación docente que efectivamente le ayudara a desarrollar sus competencias docentes digitales en clase. Las formaciones siempre son clasificadas como técnicas y que obedecen a exigencias institucionales cuanto, al tiempo, es decir, muy rápidas, pero sin eficacia. Además, vale resaltar que los entrevistados, directora y profesores no poseen formación pedagógica y siente ese hueco en su práctica docente.

La verdad es un tema complejo dentro de la dinámica de la institución donde trabajo. Les falta mucho el tema pedagógico, yo creo que falta muchísimo la formación, pero, no solamente la formación técnica sino la formación pedagógica. Y para eso, yo creo que debe ser una formación continuada, no puede ser un curso de dos días intensivo, tres días en verano. Nosotros siempre proponemos formaciones o actividades, pero ellos todavía tienen esa mentalidad de que la formación es aprender una herramienta. No es algo técnico y práctico, porque nosotros dentro de nuestra Institución, lo que hacemos es participar de formaciones de otras universidades. No se apuesta por la formación pedagógica, que es una lucha constante que tengo yo y los profesores como equipo académico y como directora, enseñar la necesidad y la importancia de esa formación. (Directora)

Más adelante, al ser cuestionada si fuera aceptable un proyecto de formación docente en la institución, ella nos deja claro tanto la demanda de ese proyecto como la dificultad de implantarlo.

iSí! Yo creo que sí, porque justamente por el hecho de que hay muy poca oferta dentro de la propia Institución. Es verdad que muchas personas son profesores porque hicieron un máster, un doctorado, pero nunca tuvieron una formación didáctica. Lo que ellos solicitan, y yo creo que es nuestra lucha, es que fuera una formación didáctica, o sea, pedagógica, que i Vale! Se enseñe una tecnología, pero, desde el punto de vista didáctico y pedagógico, que es lo que les falta a esos profesores. A nosotros, a mi también. (Directora)

Asimismo, la directora ratifica que la institución tiene recursos e instalaciones modernas y actualizadas, tales como laboratorios con herramientas de alta tecnología, softwares, equipos y espacio para trabajo y clases en ese ámbito, 
pero le falta profesores especializados, es decir con formación adecuada para utilizarlos, sobre todo desde el punto de vista pedagógico.

Un laboratorio de una alta tecnología que se podría utilizar para hacer proyectos de una manera didáctica, pero que todavía no es utilizado, o sea, el potencial que tiene no está siendo utilizado. Porque no hubo una formación adecuada hasta ahora, para realmente aprovechar muchísimo esa tecnología dentro del contexto de adaptación de la docencia. Pero esa es la cuestión, iAlli está la tecnología y el laboratorio prácticamente vacío! Entonces, eso para mí es un símbolo y es un síntoma muy fuerte de la necesidad de una formación... (Directora)

En el relato de los profesores observamos algunos contrastes con relación a las categorías establecidas: Formación inicial / Docencia / Necesidades formativas / Resistencia a la tecnología / Adquisición de las Competencias digitales y Pedagógicas / Implicaciones de la institución en ese proceso. Ambos profesores A y B no tuvieron formación docente y tampoco ninguna formación relacionada con el desarrollo de sus competencias digitales y/o docentes digitales. Fue un proceso intuitivo y autodidacta para ambos, pero constatamos que sus trayectorias son distintas, así como la manera de lidiar con el entorno digital y la tecnología propiamente dicha.

[...] no tengo una formación docente especifica en el sentido de que... yo me he preparado en mi carrera, he estudiado una carrera de Bellas Artes, que no teníamos asignaturas de Pedagogía, de la enseñanza de las Bellas Artes, por ejemplo, ni tampoco hice el CAP, como comentábamos la semana pasada, por lo tanto, mi formación docente fue autodidacta. (Profesor A)

[...] formación en competencias digitales son las que he ido adquiriendo en los últimos años, a raíz de que han ido surgiendo todo este mundo tecnológico y digital, obviamente yo por edad y mi formación, que además mi formación viene del ámbito humanístico y tal, no había nada, de hecho, yo acabé la carrera sin ordenadores, porque no existian los ordenadores. (Profesor B)

Concretamente hay un hueco en la formación docente y una brecha en las competencias digitales del profesorado, aprender intuitivamente o como autodidacta significa que el desarrollo y los avances están comprometidos considerando que, si el Profesor A tiene más habilidad y/o competencia con la tecnología, incluso desde el punto de vista técnico, que el Profesor B, su desarrollo 
será distinto y, consecuentemente, sus competencias digitales. En los relatos a seguir observamos que hay un contraste entre ambos en ese aspecto.

[...] claro hasta ahora, ha cambiado mucho y no ha cambiado tanto, es decir, por qué ha cambiado mucho, tenemos muchas cuestiones a nuestro alrededor y trabajamos y vivimos de un modo como muy digital, pero es cierto que desde mi punto de vista, muchas de estas cuestiones, o muchas de estas tareas, o muchas de estas acciones que ahora hacemos de un modo digital, las hacemos de un modo digital porque nos ponen en las manos un aparatito para que las hagamos, pero es que se pueden hacer de otra manera sin ningún problema; tampoco aporta ni mejoría ni no mejoría. A mí la tecnología me encanta, pero me encanta si me aporta algo, la tecnología por la tecnología a mí no me dice nada. (Profesor B)

Yo tenía que adaptarme más aún, porque yo tenía que adaptar también lo que estaba explicando, o sea, tenía que, por un lado, tenía que estudiar las novedades que me permitían acceder a las posibilidades. Un cambio de contenido, porque cambia el contenido, es un cambio de las herramientas de trabajo, y es un cambio, creo yo de mirada, de postura, de mentalidad. (Profesor A)

En efecto, la diferencia de punto de vista de ambos profesores es muy clara, hay una resistencia con la tecnología y la posibilidad de desarrollar las competencias digitales, a lo mejor una resistencia del principio que persiste hasta hoy.

Esta democratización del conocimiento que está muy bien, pero hay que saber. Hay que tener un filtro. Yo la impresión que tengo es que como estamos en un momento híper tecnológico, la tecnología tiene que invalidar las demás opciones, claro y dices ¿'Y por qué no pueden convivir? (Profesor B)

A continuación, en otro momento de la entrevista, habla de una manera menos resistente y más "conciliadora" con la tecnología:

Hay que tener algo que ayude al profesor a pensar en cómo utilizar, y que la tecnología venga de alguna manera también para fomentar el pensamiento crítico, porque no es incluir la tecnología en el sentido de "vamos a poner la tecnología, porque en la modernidad las cosas están así: Hay que tener tecnología. Hay que tener tecnología." iNo! sino ¿ De qué manera dentro de tu clase o con el contenido que utilizas puedes añadir una herramienta? ¿Cómo puedes hacer eso? ¿Cuál sería la mejor herramienta para este contenido? ¿Cuál sería la mejor herramienta para trabajar tales puntos de la Historia del Arte? Y eso es formación. Y eso es pensar ¿sabes? "Nadie me ha enseñado a hacer clases, ni nadie me ha enseñado a introducir la tecnología en mis 
clases; entonces claro, lo haces, porque llega un momento en que lo tienes que hacer. (Profesor B)

No obstante, el Profesor A considera que la tecnología aporta valor a sus clases, a su desarrollo profesional y al aprendizaje de sus alumnos. Conviene subrayar que su formación fue 100\% autodidacta, buscaba nuevas ideas, conocimientos y aunque no tuviera una formación pedagógica previa, siempre relacionaba lo que aprendía con la pedagogía.

Entonces pues, nada, al principio lo que pasaba es que buscaba los apuntes que yo había tomado de cuando era estudiante, y entonces luego, compraba libros y estudiaba y preparaba las clases, o sea, ya cada clase que daba pues, requería muchas horas y muchos días de trabajo, por darles el material adecuado para poder transmitir los conocimientos. Te vas preparando de manera autodidacta a medida que vas dando clases, y bueno, siempre intentando mejorar, es decir, basándome en la experiencia. (Profesor A)

Llega un momento en que yo tengo que explicar cosas que no he aprendido en la Universidad, en la escuela, tengo que explicar cosas que he aprendido por mi mismo, porque lo digital es tan nuevo, que no tiene nada que ver con todo lo que yo estudié. Lo que ocurre, que sí que es cierto, que la carrera que nosotros hacíamos de cinco años que tenía muchas asignaturas, que me han ayudado a enfocar esta cuestión tecnológica desde una perspectiva más pedagógica, porque no teníamos una asignatura de pedagogía propiamente. (Profesor A)

Con respecto a la llegada de la tecnología en la educación y por consiguiente las posibles resistencias que han ocurrido con muchos profesores, es evidente la diferencia de posicionamiento de ambos profesores. Vale resaltar que eses cambios demandaban nuevas habilidades y competencias, además de una nueva postura y actitud ante ese nuevo desafío.

Siempre he pensado que la enseñanza tiene que ser transversal. Y entonces, en ese sentido, pues, siempre he buscado la manera de mejorar el trabajo que estoy haciendo, y como profesor pues, es dar clases, obviamente. Y iClaro! En ese sentido, la tecnología fue un reto. (Profesor A)

Yo lo que vi es una posibilidad de poder hacer más cosas, es decir, que venía a sumar, esa es la impresión que a mí me dio, en todos los niveles. Que me permitía herramientas nuevas. Todo, cuanto yo hago en clase, todo lo hago con una finalidad pedagógica, y más ahora iMás que nunca! Que tengo más experiencia y qué, además, ya llevo muchísimos años dando clases durante muchas horas a la semana. (Profesor A) 
OSin embargo, ambos consideran importante y necesarias la formación docente, aunque muchas veces, por lo que se refiere a las necesidades formativas hay dificultades en identifícalas

Vale, seguramente mis necesidades formativas no las conozco, pero no las conozco por desconocimiento, ahora te explico. Todo lo que yo conozco de competencias, o sea, del ámbito digital, de todas las herramientas digitales las intento aplicar a la docencia, pero más lo que decíamos el otro día, más por una cuestión de atraer al estudiante. (Profesor B)

Por otra parte, otro punto que observamos en las entrevistas es la utilización de la tecnología está muy condicionada al nuevo perfil de alumno que se presenta en la universidad, es decir, los profesores consideran los alumnos muy digitales y visuales, así que la tecnología se convierte en una manera de atraer la atención de los alumnos.

Lo que pasa es que el cómo, es para lo que yo intento aplicar todo lo digital porque ellos funcionan de manera digital, entonces, ¿qué me faltaría?, pues seguramente tener más conocimiento de su mundo digital, entonces, si tuviese más conocimiento, seguramente tendría más herramientas. (Profesor B)

Entonces hay que adaptarse a la tecnología, pero también, a los alumnos que vienen que son más visuales, que necesitan inputs más... como te lo diría, más atractivos. Que capten su atención y que lo hagan cada cierto tiempo en clase. (Profesor A)

En cuanto a formación docente, ambos profesores reconocen su importancia y necesidad y comparten de la idea de estar vinculada a los aspectos pedagógicos, también señalado y ratificado en el relato de la directora de la institución. Por ejemplo, planificar una formación para un profesorado que posibilite el desarrollo de más competencias digitales, dentro de un entorno educativo, no se puede desvincular de las competencias pedagógicas. Porque es donde debe empezar, la competencia pedagógica, te posibilita reflexionar ante una herramienta y su finalidad educativa.

Entonces la Institución durante todo ese proceso, fue dando equipamientos, pero no daba una formación, o sea, que cada profesor empezó a utilizar esos equipamientos según le parecía adecuado...tengo la impresión de que en esta escuela no han sabido cuidar a los profesores. No los han sabido cuidar porque no han dado importancia a lo que realmente es fundamental 
en una Universidad y en un centro de enseñanza, que es una formación de calidad. (Profesor A)

[...] a mí me gustaría que la formación del profesorado tuviese más peso en la institución. (Profesor B)

[...] la cuestión de adaptarse a las herramientas digitales y a las TIC desde una perspectiva pedagógica no, no me la han enseñado en ningún sitio, y creo en ese sentido en la necesidad, porque de hecho las uso, y eso significa pues, que en algún momento he tenido que aprenderlas, y lo que me queda, de hecho, todavía. (Profesor A)

A continuación, el Profesor A analiza de manera contextualizada. Desde el punto de vista institucional la importancia de la llagada de la tecnología en la educación, así como el perfil de ese nuevo alumno que ingresa en la universidad, con nuevas demandas y un nuevo posicionamiento frente a la cultura digital.

Aquí hay que tener en cuenta que esos cambios macro estructurales que vienen de más arriba pues, más o menos a todas han afectado por igual, pero, cada institución se ha adaptado de una manera. Y yo pienso que una de las razones que han provocado los cambios que comento, es justamente el hecho de que la tecnología digital ha evolucionado, ha permitido una evolución en la comunicación y en el perfil de los estudiantes de tal manera que, bueno, desde la perspectiva de los que mandan pues, ha hecho necesario ese cambio general de lo que es la enseñanza universitaria para adaptarla a los nuevos tiempos, que está muy relacionado pues, con la era de la información y de la imagen en la que vivimos. (Profesor A)

En conclusión, la formación docente es un reto, no hay como huir, los profesores tienen necesidades formativas, tanto del punto de vista pedagógico como del punto de vista digital. Planificar un plan de formación docente priorizado la construcción de las competencias docentes digitales es condición sine qua non para el desarrollo profesional docente y para que haya avances en proceso educativo.

Vale resaltar que ambos profesores entrevistados y la directora vinculaba la tecnología a los aspectos pedagógicos, es decir, la dimensión técnica de la tecnología se hace vacía y se agota en sí misma, pierde su aspecto reflexivo y critico tan importante y exigido en la educación para que se reafirme y se asegure el valor primordial de la educación, la transformación social.

Para finalizar, específicamente en lo que se refiere a la Educación a Distancia, 
el valor del pedagógico y la labor para que esté intrínsecamente vinculado a la tecnología en se hacen más presentes, dado que la tecnología es el punto clave de conexión de las herramientas con las dimensiones pedagógicas y educativas.

Por consiguiente, plantear una formación docente en la Educación a Distancia, priorizando la construcción de las competencias docentes digitales requiere un estudio detallado de las necesidades formativas de ese profesorado, tanto del punto de vista digital como pedagógico, considerando el entorno institucional, las políticas públicas vigentes y el espacio virtual elegido, visto que hay mucha oferta en el mercado. Así pues, es importante tener un objetivo claro de lo que se propone, es decir: ¿ Por qué quiero implantar la Educación a distancia? ¿Cuáles herramientas serán utilizadas? ¿Cuáles objetivos Pedagógicos? ¿Cómo voy a seleccionar y formar el profesorado?

Para finalizar, ratificamos que hay que tener en cuenta esas cuestiones, su importancia en el momento de pensar en la formación docente y la construcción de las competencias docentes digitales en el entorno de EaD. Por otro lado, la docencia exige compromiso, afecto, deseo de transformar y eso hace toda la diferencia, el espacio formativo es un espacio de construir y compartir conocimientos, de donarse, de cambio de actitud y de valorarse como profesional.

[...] lo primero que pensé, pues eso, buscar un trabajo dentro de la enseñanza porque, aunque no lo había pensado en un principio, sí que me parecía... me atraía, me gustaba la idea de poder formar personas. En cambio, la enseñanza para mí tiene un valor extra, y luego el hecho de que te llena, de que tú estás en contacto con gente, y gente que se renueva cada año. (Profesor A)

Por tanto, para construir un plan o modelo de formación que integre las TIC y promueva el desarrollo profesional docente hay que considerar las necesidades formativas actuales y futuras a través de un diagnóstico detallado y preciso.

Para mí, este concepto incluye el de la formación del profesorado, tanto inicial como permanente, como un proceso dinámico y evolutivo de la profesión y función docente [...]. Este desarrollo engloba los procesos que mejoran el conocimiento profesional, las habilidades y las actitudes [...]. El desarrollo profesional del profesorado empieza en su entrada en los estudios que le habilitarán para la profesión, lo que nos conduce a afirmar que el desarrollo profesional del profesorado es uno, pero con diferentes etapas. (IMBERNÓN, 1994). 
En efecto, es importante tener en cuenta los conceptos abordados y los aspectos citados para pensar en construir un Plan o modelo de formación que integre la tecnología y contemple la adquisición de las competencias digitales promoviendo el desarrollo profesional docente, respectando los distintos contextos culturales y sus respectivos marcos referenciales. Además, hay que considerar que en el entorno universitario hay una diversidad de formaciones y muchos son especialistas con carencia de formación pedagógica.

Así pues, hay pensar en una propuesta formativa que priorice un enlace entre la tecnología y los aspectos pedagógicos, es decir, pensar en la construcción de las competencias docentes digitales a partir de la dimensión educativa.

\section{REFERENCIAS}

CORTÉS, P. El sentido de las historias de vida en investigaciones socio educativas. Una revisión- crítica. In: HERNÁNDEZ, F.; SANCHO, J. M.; RIVAS, J. I. (Coord.). Historias de vida en educación: biografías en contexto. Barcelona: Esbrina, 2011. p. 68-74. Disponibre en: http://hdl.handle.net/2445/15323

FERRARI, A. Digital competence in practice: an analysis of frameworks. Luxembourg: Publications Office of the European Union, 2012.

IMBERNÓN, F. La formación y el desarrollo profesional del profesorado. Hacia una nueva cultura profesional. Barcelona: Graó, 1994.

MEDINA, L. Delimitacion teórica y conceptual de la formación docente en la universidad publica mexicana. In: MEDINA, L; BELTRÁN, E. E. S. Los servicios educativos en la formación de profesores universitarios: el caso de la UAEM. Ciudade del Mexico: Universidad Autonoma del Estado de Mexico, 2006.

POZOS, K. Evaluación de necesidade de formación continua en competencia digital de profesorado universitario mexicano para la sociedad del conocimiento. 2015. Tesis (Doctorado en Calidad y Procesos de Innovación Educativa)- Universitat Autònoma de Barcelona (UAB), Bellaterra, España, 2015.

SANCHO, J. M.; HERNÁNDEZ, F. (Ed.). Tecnologias para transformar a educação. Porto Alegre: Artmed, 2006.

TEJADA, J. Competencias docentes. Profesorado. Revista de Currículum y Formación del Profesorado, Granada, España, v. 13, n. 2, p. 1-15, ago. 2009. 
UNESCO. Construir sociedades del conocimiento. 2018. Disponible en: https://es.unesco. org/themes/construir-sociedades-del-conocimiento. Aceso en: 10 abr. 2019.

UNESCO. Competency standards modules. ICT competency standards for teachers. 2008. Disponible en: http://unesdoc.unesco.org/images/0015/001562/156207e.pdf. Aceso en: 10 abr. 2019.

ZABALZA, M. A. Competencias docentes del profesorado universitario. Calidad y desarrollo profesional. Madrid: Narcea, 2007.

\section{Sobre os autores:}

Isamélia Santos Guimarães Carvalho: Doctoranda en Educación por la Facultad de Ciencias de la Educación - Universidad Autónoma de Barcelona. DEA en Comunicación y Periodismo y Máster en Comunicación y Educación, ambos por la Universidad Autónoma de Barcelona (UAB). Licenciada en Pedagogía con posgrado en Psicopedagogía y Formación en Psicoanálisis. Foco en enseñanza superior, formación de profesores, consultoría y orientación pedagógica, así como a la aplicación de las TICs a la educación. Escritora y productora de materiales didácticos, así como colaboradora en distintas Universidades. Ha actuado como Profesora y Formadora de la Educación Primaria y Educación Superior, dedicándose a las asignaturas de Psicología de la Educación y Psicología del Aprendizaje. Igualmente ha sido Profesora Gestora de ambas asignaturas, coordinando el grupo de profesores y los contenidos del currículo pedagógico. En la actualidad investiga acerca de Formación Docente y la adquisición de las competencias docentes digitales. Planifica y desarrolla trabajos de formación docente con distintas instituciones de educación. E-mail: isacarvalho27@gmail.com, Orcid: http://orcid.org/0000-0002-8005-525X

José Tejada: Licenciado en Psicología y en Pedagogía, Doctor en Ciencias de la Educación por la Universidad Autónoma de Barcelona, es Catedrático de Didáctica y Organización Escolar. Está adscrito al Departamento de Pedagogía Aplicada de la Universidad Autónoma de Barcelona. Especialista en evaluación de programas, innovación formativa, formación para el trabajo y formación de profesionales de la formación, ha ejercido su actividad docente e investigadora universitaria y extrauniversitaria en estos campos, tanto en licenciatura, doctorados, másteres y 
postgrados, nacionales e internacionales. Es miembro del Grupo CIFO (Colectivo de investigación en Formación Ocupacional). Forma parte de una veintena de Consejos Científicos y Editoriales de Revistas especializadas en el ámbito de las Ciencias de la Educación en España y otros países. En la actualidad es Director de la Revista EDUCAR (Servició Publicaciones de la UAB). E-mail: jose.tejada@uab. cat, Orcid: http://orcid.org/0000-0001-9044-8826

Katia Verónica Pozos Pérez: Doctora en Educación especialista en Educación y Nuevas Tecnologías, formación del profesorado en competencia digital, así como en investigación en educación social de base comunitaria a nivel local, nacional, europeo e internacional, colaborando activamente para el Centro UNESCO de Cataluña, la Dirección General de Juventud, el Instituto Municipal de Educación de Barcelona, la Comisión Europea, Televisión de Cataluña, Fundación Obra Social La Caixa, Departament d'Ensenyament de la Generalitat de Catalunya, entre otros. E-mail: katiaveronica.pozos@uab.cat, Orcid: http:// orcid.org/0000-0003-2780-4538

\section{Recibido en marzo de 2019}

Aprobado en agosto de 2019 
